\title{
Theory of second harmonic generation in randomly oriented species
}

\author{
David L. Andrews *, Philip Allcock, Andrey A. Demidov ${ }^{1}$ \\ School of Chemical Sciences, University of East Anglia, Norwich NR4 7TJ UK
}

Received 7 June 1994

\begin{abstract}
It is well known that second harmonic generation (SHG) is a process forbidden within atomic and molecular fluids. Nonetheless recent experimental observations of second harmonic evolution in suspensions of randomly oriented Halobacterium halobium purple membranes have raised new questions about the precise criteria which determine prohibition of the second harmonic. To address the problem a theoretical framework for SHG is developed that specifically deals with molecular systems, and is therefore cast in terms of molecular properties with more regard to the influence of the local structure. This contrasts with the classical approach based on bulk susceptibilities, which has not proved adequate to explain the conflicting experimental results. By properly formulating the detailed procedure for dealing with the necessary orientational averages, the present theory discloses a relationship between the coherent process of second harmonic generation and a directed component of its incoherent counterpart, hyper-Rayleigh scattering. Inter alia, the theory explains the SHG detected in purple membrane suspensions. The polarisation features of the harmonic evolution are also considered more generally, and in particular it is shown that the SHG signal will persist under conditions of circularly polarised pumping. This specific polarisation feature will allow experimental validation of the theory.
\end{abstract}

\section{Introduction}

In the literature there is overwhelming written evidence, based both on experimental and theoretical studies, to refute the possibility of generating an optical second harmonic on the passage of light through isotropic media. For this reason the technique of second harmonic generation (SHG) has acquired important analytical applications as a sensitive means of probing fluid surfaces. Exceptions to the forbidden character of even harmonics in fluids or otherwise microscopically disordered media arise only under special circumstances, as listed in a recent review [1]. One is where there is an induced anisotropy, as for example may be conferred on a polar fluid by application of a static electric field [2-5]. A higher-order process entailing the coupling of harmonic conversions can result in production of an even harmonic through a mechanism involving an even number of electric dipole interactions [6]. It has also been suggested that the explanation for the observation of weak second harmonic signals gradually evolving in glass fibres is the formation of a grating or spatially periodic electric field, effectively destroying the

\footnotetext{
* Corresponding author.

' Permanent address: Quantum Radiophysics Division, Physics Department, Moscow State University, Lenin Hills, 119899 Moscow. Russia.
} 
symmetry of the bulk [7-11]. Indeed in these cases it is hard to rationalise the evolution of a second harmonic in terms of anything other than a structural effect.

It is the subject of considerable interest that second harmonic evolution has recently been shown to occur in suspensions of randomly oriented units of Halobacterium halobium purple membranes (PM) [12]. The optical properties of this system for the fundamental and harmonic frequencies employed are dominated by the bacteriorhodopsin (BR) pigment, which has a specific photobiological property of pumping protons across the cell membrane. This process is initiated through light absorption by a specific chromophore: retinal, bound to the protein molecule [13]. The process of proton pumping leads to the conversion of light to electrochemical energy through the formation of separated charges, and hence ultimately the formation of ATP. One can find recent reviews of the structure of BR in Refs. [14,15] and of its properties in Refs. [16,17].

In this paper a theory is developed which, by proper differentiation of orientational and distributional averages, clarifies the distinction between the behaviour of molecular fluids and other systems comprising randomly oriented species. In contrast to fluids in which there can arise a second harmonic signal through a statistical correlation between the time-varying orientations of molecules within a certain distance, as for example through dipole-dipole interaction [18,19], we here consider other systems which embody a degree of essentially time-independent, structurally determined, orientational order. Examples include structured aggregates of molecular units as for example in membranes, and partially ordered solids in which orientational order extends over local domains, as in certain glasses. To draw comparisons and distinctions between particular physical cases, the theoretical description developed in Section 2 is addressed first to an ideal molecular fluid, and then to a randomly oriented ensemble of identically structured aggregates referred to as units. The method to be presented is of course amenable to modification, by incorporating appropriate distribution functions, for application to more complex systems in which different units have diverse structures or contain different numbers of molecules.

Once a relation is established between the coherent process of second harmonic generation and its incoherent counterpart termed hyper-Rayleigh scattering, features of the polarisation behaviour associated with the directed component are considered in Section 3. It is shown that one specific detail relating to the polarisation of the pump radiation affords a straightforward method of experimentally validating the proposed theory. Finally in Section 4 , an overview of the general results is followed by detailed consideration of how the new theory specifically relates to the experiments on bacteriorhodopsin. It is shown that the results are consistent with an interference of quantum probability amplitudes for harmonic conversion by individual bacteriorhodopsin molecules within the membrane.

\section{Orientational and distributional averages}

In molecular systems it is the individual molecular centres or chromophores that are responsible for harmonic emission, and account must be taken of the distribution of their orientations [20]. Consider a process in which pairs of incident photons of wave-vector $\boldsymbol{k}$ are converted to second harmonic photons of wave-vector $\boldsymbol{k}^{\prime}$. The radiant intensity $\mathscr{I}$ of second harmonic emission by an assembly of $N$ molecules individually labelled $\xi$ is expressible in the form [1]

$$
\mathscr{I}=\mathscr{D}^{(2)}\left|\sum_{\xi}^{N}\left(\boldsymbol{\beta}_{\xi}: \bar{e}^{\prime} e \boldsymbol{e}\right) \exp \left(\mathrm{i} \Delta k \cdot \boldsymbol{R}_{\xi}\right)\right|^{2},
$$

where $\mathscr{D}^{(2)}$ is a beam parameter given by

$$
\mathscr{D}^{(2)} \approx \frac{k^{\prime 4} I_{\omega D_{\omega}^{2}}^{(2)}}{32 \pi^{2} c \epsilon_{0}^{3}},
$$

$\omega$ being the frequency of the laser pump, $I_{\omega}$ its intensity (power per unit cross-section) and $g_{\omega}^{(2)}$ the degree of second order coherence. In Eq. (1), $\boldsymbol{\beta}_{\xi}$ is the hyperpolarisability tensor, the structure of which is discussed in Section $4, e$ 
and $\boldsymbol{e}^{\prime}$ are the pump (fundamental) and harmonic polarisation vectors, and the exponential term contains the molecular position vector $\boldsymbol{R}_{\xi}$, relative to an arbitrary fixed origin and the wave-vector mismatch $\Delta \boldsymbol{k}=2 \boldsymbol{k}-\boldsymbol{k}^{\prime}$. Both the hyperpolarisability and the polarisation vectors are assumed to be complex, so as to accommodate near-resonance behaviour and circular polarisations, respectively. The complex conjugate of $\boldsymbol{\beta}_{\xi}$ is denoted with an asterisk, that of either polarisation vector with an overbar.

\section{J. Background; treatment of ideal molecular fluids}

Since the summation over molecules in (1) appears inside the modulus square, the intensity of harmonic emission contains $N^{2}$ terms, of which $N$ are diagonal (single centre) contributions and the other $\left(N^{2}-N\right)$ off-diagonal. The former diagonal terms are expressible as $N$ times the orientationally averaged result for a single molecule:

$$
\sum_{\xi}^{N}\left|\boldsymbol{\beta}_{\xi}: \overline{\boldsymbol{e}}^{\prime} \boldsymbol{e e}\right|^{2}=N\left\langle\left(\boldsymbol{\beta}_{\xi}: \overline{\boldsymbol{e}}^{-} \boldsymbol{e} \boldsymbol{e}\right)\left(\boldsymbol{\beta}_{\xi}^{*}: \boldsymbol{e}^{\prime} \overline{\boldsymbol{e} \boldsymbol{e}}\right)\right\rangle=N\left\langle\boldsymbol{\beta}_{\xi} \boldsymbol{\beta}_{\xi}^{*}:: \overline{\boldsymbol{e}}^{-} \boldsymbol{e} \boldsymbol{e} \boldsymbol{e}^{\prime} \overline{\boldsymbol{e} \boldsymbol{e}}\right\rangle,
$$

and as such are associated with incoherent harmonic emission, the relatively weak process of hyper-Rayleigh scattering [21]. At this stage it is worth noticing that, since the phase factor disappears, the result applies irrespective of the value of $\Delta k$.

Three important features can immediately be identified in the result (3). One is the obvious but significant fact that incoherent scattering depends linearly on the number of scatterers $N$, and therefore delineates the process as colligative. Secondly, the rotational average in (3) is taken over the modulus square of the matrix element $\left(\boldsymbol{\beta}_{\xi}^{\mathrm{f}}: \bar{e}^{\prime} e e\right)$. Thirdly, it may be noted that hyper-Rayleigh emission usually extends in all directions, as witnessed by the lack of the directive phase factor. In all respects, these features mark important differences from the coherent term to be examined next.

Under circumstances where the individual molecules are orientationally uncorrelated, the off-diagonal terms are expressible through

$$
\sum_{\xi}^{N} \sum_{\xi^{\prime}}^{N-1}\left\langle\left(\boldsymbol{\beta}_{\xi}: \overline{\boldsymbol{e}}^{\prime} \boldsymbol{e} \boldsymbol{e}\right)\right\rangle\left\langle\left(\boldsymbol{\beta}_{\xi^{\prime}}^{*}: \boldsymbol{e}^{\prime} \overline{\boldsymbol{e} \boldsymbol{e}}\right)\right\rangle \exp \left(\mathrm{i} \Delta \boldsymbol{k} \cdot \boldsymbol{R}_{\xi \xi^{\prime}}\right),
$$

which is an interference term dependent, through the phase factor $\exp \left(\mathrm{i} \Delta \boldsymbol{k} \cdot \boldsymbol{R}_{\xi \xi^{\prime}}\right)$, on the relative displacement, $\boldsymbol{R}_{\xi \xi^{\prime}} \equiv \boldsymbol{R}_{\xi}-\boldsymbol{R}_{\xi^{\prime}}$, of each pair of molecules with respect to the wave-vector mismatch, $\Delta \boldsymbol{k}$. The expression (4) therefore represents a coherent signal for the second harmonic. Since in an ideal fluid the spatial and orientational coordinates of each molecule are uncorrelated, the above expression can be written as

$$
\left(\eta_{N}-N\right)\left|\left\langle\left(\boldsymbol{\beta}_{\xi}: \overline{\boldsymbol{e}}^{\prime} \boldsymbol{e} \boldsymbol{e}\right)\right\rangle\right|^{2},
$$

where

$$
\eta_{N}=\left|\sum_{\xi}^{N} \exp \left(\mathrm{i} \Delta \boldsymbol{k} \cdot \boldsymbol{R}_{\xi}\right)\right|^{2}
$$

is a coherence factor. As shown elsewhere [22], the case of perfect phase-matching $\Delta k=0$, together with the assumption of a completely random spatial distribution for the molecular displacement vectors $\boldsymbol{R}_{\xi}$, leads to the simple result

$$
\eta_{N}=N^{2}(\Delta k=0)
$$

so that the response given by Eq. (5) entails a quadratic dependence on the number of scatterers. More generally, for $\Delta k \cong 0$, we obtain the well-known $\operatorname{sinc}^{2}$ dependence 


$$
\eta_{N}=N^{2} \frac{\sin ^{2}\left(\frac{1}{2}|\Delta k| L\right)}{\left(\frac{1}{2}|\Delta k| L\right)^{2}}
$$

on the propagation distance $L$.

Calculation of the tensor orientational averages in Eqs. (3) and (5) necessitates more detailed analysis of the inner product $\boldsymbol{\beta}: \bar{e}^{-} \boldsymbol{e} e$ which can be written as

$$
\boldsymbol{\beta}: \bar{e}^{\prime} e \boldsymbol{e}=\beta_{i_{1} i_{2} i_{3}}\left(\bar{e}_{i_{1}}{ }^{\prime} e_{i_{2}} e_{i_{3}}\right) \text {, }
$$

with components referred to a reference frame in which the radiation tensor components are fixed, and the molecular nonlinear susceptibility components thus necessarily vary with molecular orientation. Note that the structure of the hyperpolarisability tensor is such that it is symmetric with respect to interchange of its last two indices, i.e. $\beta_{i_{11} i_{2} i_{3}}=\beta_{i_{1} i_{i 2}}$, where $i_{1}, i_{2}$ and $i_{3}$ each can represent $x, y$ or $z$. It is generally more convenient to refer the molecular tensor to a molecule-fixed frame, denoted below by indices $\lambda_{r}$ in terms of which its own components are rotationinvariant. We then have

$$
\boldsymbol{\beta}: \bar{e}^{\prime} e \boldsymbol{e}=\beta_{\lambda_{1} \lambda_{2} \lambda_{3}}\left(\bar{e}_{i_{1}}{ }^{\prime} e_{i_{2}} e_{i_{3}}\right) l_{i_{1} \lambda_{1}} l_{i_{2} \lambda_{2}} l_{i 3 \lambda_{3}},
$$

where only the direction cosines $l_{i_{r} \lambda_{r}}$ vary with molecular rotation. The orientational averages in (3) and (5) are thus obtained by averaging over the direction cosine products;

$$
\begin{aligned}
& \left\langle\left|\boldsymbol{\beta}: \bar{e}^{\prime} \boldsymbol{e} e\right|^{2}\right\rangle=\beta_{\lambda_{1} \lambda_{2} \lambda_{3}} \beta_{\lambda_{4} \lambda_{5} \lambda_{6}}^{*}\left(\bar{e}_{i_{1}}{ }^{\prime} e_{i_{2}} e_{i_{3}}\right)\left(e_{i_{4}}{ }^{\prime} \bar{e}_{i s} \bar{e}_{i 6}\right)\left\langle l_{i_{11} \lambda_{1}} l_{i 2 \lambda_{2}} l_{i 3 \lambda_{3}} l_{i 4 \lambda_{4}} l_{i s \lambda s} l_{i 6 \lambda_{6}}\right\rangle, \\
& \left|\left\langle\boldsymbol{\beta}: \bar{e}^{\prime} e \boldsymbol{e}\right\rangle\right|^{2}=\left|\beta_{\lambda_{1} \lambda_{2} \lambda_{3}}\left(\bar{e}_{i_{1}}{ }^{\prime} e_{i_{2}} e_{i_{3}}\right)\left\langle l_{i_{1} \lambda_{1}} l_{i_{2} \lambda_{2}} l_{i 3 \lambda_{3}}\right\rangle\right|^{2} \text {. }
\end{aligned}
$$

The result of applying the rotational average in (12) is that the product vanishes, because it leads to a vector product of the polarisation vector $e$ with itself [20]. It is for this reason that second harmonic generation is a process forbidden within the bulk of an ideal fluid.

\subsection{Macromolecules, suspensions and partially ordered solids}

Suppose we now have an assembly of $M$ randomly oriented units labelled $u$, each containing $n$ discrete molecules. It is assumed that within each unit these molecules have a structurally imposed orientational correlation. Starting again from basic principles, the intensity of harmonic emission is explicitly given by the following equation, expressed in a form which identifies the collective response of each unit:

$$
\mathscr{I}=\mathscr{D}^{(2)}\left|\sum_{u}^{m}\left(\boldsymbol{\beta}_{(u)}: \overline{e^{\prime}} \boldsymbol{e} \boldsymbol{e}\right) \exp \left(\mathrm{i} \Delta \boldsymbol{k} \cdot \boldsymbol{R}_{u}\right)\right|^{2} \text {, }
$$

with $\boldsymbol{R}_{u}$ the position vector for the origin of an arbitrary Cartesian frame of reference having a fixed orientation within unit $u$, and where

$$
\beta_{(u) i_{1} i_{2 i} i_{3}}=\sum_{\xi}^{n} \beta_{(\xi) \lambda_{1} \lambda_{2} \lambda_{3}} l_{i_{1} \lambda_{1}}^{\xi} l_{i_{2} \lambda_{2}}^{\xi} l_{i_{3} \lambda_{3}}^{\xi} \exp \left[\mathrm{i} \Delta \boldsymbol{k} \cdot\left(\boldsymbol{R}_{\xi}-\boldsymbol{R}_{u}\right)\right] .
$$

The latter intra-unit summation accommodates the hyperpolarisabilities, each with its own relative position and orientation referred to the common Cartesian frame, of all the molecular components.

Adding the harmonic signals associated with each distinct unit, assuming that the various units have a random distribution of orientations with respect to the radiation, and that the concentration of the disperse phase is sufficiently low that any correlation between molecules of different units can be neglected, we obtain

$$
\mathscr{I}=\mathscr{I}_{1}+\mathscr{I}_{2}
$$


where

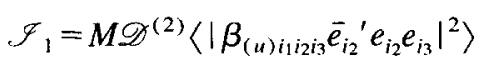

and

$$
\mathscr{I}_{2}=\left(\eta_{M}-M\right) \mathscr{D}^{(2)}\left|\left\langle\beta_{(u) i_{i 2 i} i_{3}} \bar{e}_{i_{1}}{ }^{\prime} e_{i_{2}} e_{i_{3}}\right\rangle\right|^{2} \text {. }
$$

The coherence factor $\eta_{M}$ in (17), defined in a similar way to (6), here relates to the spatial distribution of units within the system and thus once again factorises out from the unit orientational average. In this case it is no longer appropriate simply to use the terms "incoherent" and "coherent" as designations for the two contributions, $\mathscr{I}_{1}$ and $\mathscr{I}_{2}$, (see below). Certainly the latter, which represents constructive interference between different units, has an intrinsic dependence on polarisation that, as with (5) and (12), leads to this term vanishing.

The harmonic contribution $\mathscr{J}_{1}$ clearly has very different attributes. Most obvious is that it is associated with an orientational average over the modulus square of the unit response, giving a different polarisation behaviour. The interference between signals associated with each pair of molecules $\xi$ and $\xi^{\prime}$ within each unit generates an intensity contribution determined through (14) by

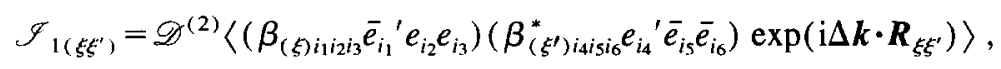

in which both the molecular nonlinear susceptibilities and also the vector $\boldsymbol{R}_{\xi \xi^{\prime}}$ have a fixed mutual orientation. Consequently the average must here be effected with regard to the orientations of these quantities relative not only to the radiation tensor $\overline{\boldsymbol{e}}^{\prime} \boldsymbol{e} \boldsymbol{e}$ but also $\Delta \boldsymbol{k}$, and this phase factor cannot in general be factorised out. As a result $\mathscr{F}_{1\left(\xi \xi^{\prime}\right)}$ averages to zero for $\xi \neq \xi^{\prime}$ except where $\Delta k \approx 0$; maximum harmonic emission clearly occurs where the pump and harmonic waves propagate collinearly, and we then have

$$
|\Delta k|=\left|2 k-k^{\prime}\right|=(2 \omega / c)\left(n_{\omega^{\prime}}-n_{\omega^{\prime}}\right),
$$

where $n_{\omega}$ and $n_{\omega^{\prime}}$ are the refractive indices for the pump and harmonic frequencies, respectively $\left(\omega^{\prime}=2 \omega\right)$. This is the origin of the directed character of the harmonic emission.

Under such circumstances the individual molecular susceptibilities are, according to Eq. (14), essentially additive within the region over which coherence is sustained. Generally this may be regarded as the range of distances which satisfy the condition $\left|\boldsymbol{R}_{\xi \xi^{\prime}}\right| \leq l_{\mathrm{c}}$, where $l_{\mathrm{c}}$ is the coherence length ${ }^{2}$ given by $\pi /|\Delta \boldsymbol{k}|$. If the dimension of each unit is substantially smaller than this distance, we obtain a simple quadratic dependence of $\mathscr{I}_{1}$ on $n$. Hence $\mathscr{F}_{1}$ can properly reflect a response that is coherent, in the sense that it arises from constructive interference between signals from various molecules within each unit. The result (16) nonetheless scales linearly with $M$, the number of such units.

\section{Polarisation behaviour}

The sixth-rank rotational average comprises products of Kronecker delta triplets, each of which is referred to either the laboratory or the molecular frame [23]. The laboratory frame deltas in particular contract with the polarisation vector components to give scalar products among $\boldsymbol{e}, \overline{\boldsymbol{e}}, \boldsymbol{e}^{\prime}, \overline{\boldsymbol{e}}^{\prime}$. From (16), the full result for the intensity of second harmonic generation in the units with ordered chromophores is thus as follows,

\footnotetext{
${ }^{2}$ For harmonic generation in solid media the propagation distance $L$ rather than $\left|\boldsymbol{R}_{\xi \xi^{*}}\right|$ features in the same type of inequality.
} 


$$
\begin{aligned}
& \mathscr{I}^{(\mathrm{SHG})}=\frac{M \mathscr{D}^{(2)}}{105}\left\{\left[11\left(\overline{\boldsymbol{e}}^{-} \cdot \boldsymbol{e}\right)\left(\boldsymbol{e} \cdot \boldsymbol{e}^{\prime}\right)(\overline{\boldsymbol{e}} \cdot \overline{\boldsymbol{e}})-6\left(\overline{\boldsymbol{e}}^{\prime} \cdot \boldsymbol{e}\right)\left(\boldsymbol{e}^{\prime} \cdot \overline{\boldsymbol{e}}\right)-5(\boldsymbol{e} \cdot \boldsymbol{e})(\overline{\boldsymbol{e}} \cdot \overline{\boldsymbol{e}})\right.\right. \\
& \left.+4\left(\overline{\boldsymbol{e}}^{\prime} \cdot \overline{\boldsymbol{e}}\right)(\boldsymbol{e} \cdot \boldsymbol{e})\left(\boldsymbol{e}^{\prime} \cdot \overline{\boldsymbol{e}}\right)-6\left(\overline{\boldsymbol{e}}^{\prime} \cdot \bar{e}\right)\left(\boldsymbol{e} \cdot \boldsymbol{e}^{\prime}\right)+4\right] \beta_{(u) \lambda \lambda \mu} \beta_{(u) \mu \nu \nu}^{*} \\
& +\left[-6\left(\overline{\boldsymbol{e}}^{\prime} \cdot \boldsymbol{e}\right)\left(\boldsymbol{e} \cdot \boldsymbol{e}^{\prime}\right)(\overline{\boldsymbol{e}} \cdot \overline{\boldsymbol{e}})+16\left(\overline{\boldsymbol{e}}^{\prime} \cdot \boldsymbol{e}\right)\left(\boldsymbol{e}^{\prime} \cdot \overline{\boldsymbol{e}}\right)+4(\boldsymbol{e} \cdot \boldsymbol{e})(\overline{\boldsymbol{e}} \cdot \overline{\boldsymbol{e}})-6\left(\overline{\boldsymbol{e}}^{\prime} \cdot \overline{\boldsymbol{e}}\right)(\boldsymbol{e} \cdot \boldsymbol{e})\left(\boldsymbol{e}^{\prime} \cdot \overline{\boldsymbol{e}}\right)\right. \\
& \left.+2(\bar{e} \cdot \bar{e})\left(e \cdot e^{\prime}\right)-6\right] \beta_{(u) \lambda \lambda \mu} \beta_{(u) \nu \nu \mu}^{*} \\
& +\left[-5\left(\overline{e^{\prime}} \cdot \boldsymbol{e}\right)\left(\boldsymbol{e} \cdot \boldsymbol{e}^{\prime}\right)(\overline{\boldsymbol{e}} \cdot \overline{\boldsymbol{e}})+4\left(\overline{e^{\prime}} \cdot \boldsymbol{e}\right)\left(\boldsymbol{e}^{\prime} \cdot \overline{\boldsymbol{e}}\right)+8(\boldsymbol{e} \cdot \boldsymbol{e})(\overline{\boldsymbol{e}} \cdot \overline{\boldsymbol{e}})-5\left(\overline{\boldsymbol{e}}^{\prime} \cdot \overline{\boldsymbol{e}}\right)(\boldsymbol{e} \cdot \boldsymbol{e})\left(\boldsymbol{e}^{\prime} \cdot \overline{\boldsymbol{e}}\right)\right. \\
& \left.+4\left(\overline{e^{\prime}} \cdot \bar{e}\right)\left(\boldsymbol{e} \cdot \boldsymbol{e}^{\prime}\right)-5\right] \beta_{(u) \lambda \mu \mu} \beta_{(u) \lambda \nu \nu}^{*} \\
& +\left[4\left(\overline{\boldsymbol{e}}^{\prime} \cdot \boldsymbol{e}\right)\left(\boldsymbol{e} \cdot \boldsymbol{e}^{\prime}\right)(\overline{\boldsymbol{e}} \cdot \overline{\boldsymbol{e}})-6\left(\overline{\boldsymbol{e}}^{\prime} \cdot \boldsymbol{e}\right)\left(\boldsymbol{e}^{\prime} \cdot \overline{\boldsymbol{e}}\right)-5(\boldsymbol{e} \cdot \boldsymbol{e})(\overline{\boldsymbol{e}} \cdot \overline{\boldsymbol{e}})+4\left(\overline{\boldsymbol{e}}^{\prime} \cdot \overline{\boldsymbol{e}}\right)(\boldsymbol{e} \cdot \boldsymbol{e})\left(\boldsymbol{e}^{\prime} \cdot \overline{\boldsymbol{e}}\right)\right. \\
& \left.-6\left(\bar{e}^{\prime} \cdot \bar{e}\right)\left(e \cdot e^{\prime}\right)+11\right] \beta_{(u) \lambda \mu \nu} \beta_{(u) \lambda \mu \nu}^{*} \\
& +\left[4\left(\bar{e}^{\prime} \cdot e\right)\left(e \cdot e^{\prime}\right)(\bar{e} \cdot \bar{e})-6\left(\bar{e}^{\prime} \cdot e\right)\left(e^{\prime} \cdot \bar{e}\right)-5(e \cdot e)(\bar{e} \cdot \bar{e})+11\left(\overline{e^{\prime}} \cdot \bar{e}\right)(e \cdot e)\left(e^{\prime} \cdot \bar{e}\right)\right. \\
& \left.-6\left(\bar{e}^{\prime} \cdot \bar{e}\right)\left(e \cdot e^{\prime}\right)+4\right] \beta_{(u) \lambda \mu \mu} \beta_{(u) \nu \nu \lambda}^{*} \\
& +\left[-6\left(\bar{e}^{\prime} \cdot e\right)\left(\boldsymbol{e} \cdot \boldsymbol{e}^{\prime}\right)(\overline{\boldsymbol{e}} \cdot \overline{\boldsymbol{e}})+2\left(\overline{\boldsymbol{e}}^{\prime} \cdot \boldsymbol{e}\right)\left(\boldsymbol{e}^{\prime} \cdot \overline{\boldsymbol{e}}\right)+4(\boldsymbol{e} \cdot \boldsymbol{e})(\overline{\boldsymbol{e}} \cdot \overline{\boldsymbol{e}})-6\left(\overline{\boldsymbol{e}}^{\prime} \cdot \bar{e}\right)(\boldsymbol{e} \cdot \boldsymbol{e})\left(\boldsymbol{e}^{\prime} \cdot \bar{e}\right)\right. \\
& \left.\left.+16\left(\bar{e}^{\prime} \cdot \bar{e}\right)\left(e \cdot e^{\prime}\right)-6\right] \beta_{(u) \lambda \mu \nu} \beta_{(u) \mu \nu \lambda}^{*}\right\},
\end{aligned}
$$

where use has been made of the symmetry in the last two indices of the hyperpolarisability tensor. The result (20) has the same polarisation structure as hyper-Rayleigh scattering ${ }^{3}$ though for non-collinear pump and harmonic radiation, where the wave-vector mismatch is such that there is no longer constructive interference within each unit, the unit hyperpolarisability tensor $\boldsymbol{\beta}_{(\mathrm{u})}$ would effectively be replaced by the molecular tensor, and $M$ by $N$.

From the general equation (20), specific results may be obtained for the intensity of harmonic emission in particular polarisation configurations. For example if the harmonic is resolved for a component with the same plane polarisation as incident plane-polarised pump radiation, the result is

$$
\begin{aligned}
& \mathscr{I}^{\mathrm{SHG}}(\perp \rightarrow \perp)=\frac{M \mathscr{D}^{(2)}}{105}\left(2 \beta_{(u) \lambda \lambda \mu} \beta_{(u) \mu \nu \nu}^{*}+4 \beta_{(u) \lambda \lambda \mu} \beta_{(u) \nu \nu \mu}^{*}+\beta_{(u) \lambda \mu \mu} \beta_{(u) \lambda \nu \nu}^{*}+2 \beta_{(u) \lambda \mu \nu} \beta_{(u) \lambda \mu \nu}^{*}\right. \\
& \left.\quad+2 \beta_{(u) \lambda \mu \mu} \beta_{(u) \nu \nu \lambda}^{*}+4 \beta_{(u) \lambda \mu \nu} \beta_{(u) \mu \nu \lambda}^{*}\right) .
\end{aligned}
$$

For a component of the harmonic rotated through $90^{\circ}$ relative to the pump polarisation plane we find a non-zero contribution given by

$$
\begin{aligned}
& \mathscr{F}^{\mathrm{SHG}}(\perp \rightarrow \|)=\frac{M \mathscr{D}^{(2)}}{105}\left(-\beta_{(u) \lambda \lambda \mu} \beta_{(u) \mu \nu \nu}^{*}-2 \beta_{(u) \lambda \lambda \mu} \beta_{(u) \nu \nu \mu}^{*}+3 \beta_{(u) \lambda \mu \mu} \beta_{(u) \lambda \nu \nu}^{*}+6 \beta_{(u) \lambda \mu \nu} \beta_{(u) \lambda \mu \nu}^{*}\right. \\
& \left.-\beta_{(u) \lambda \mu \mu} \beta_{(u) \nu \nu \lambda}^{*}-2 \beta_{(u) \lambda \mu \nu} \beta_{(u) \mu \nu \lambda}^{*}\right) .
\end{aligned}
$$

The net harmonic intensity is simply the sum of the contributions (21) and (22), i.e.

$$
\begin{aligned}
& \mathscr{I}_{\text {tot(plane) }}^{\mathrm{SGH}}=\frac{M \mathscr{D}^{(2)}}{105}\left(\beta_{(u) \lambda \lambda \mu} \beta_{(u) \mu \nu \nu}^{*}+2 \beta_{(u) \lambda \lambda \mu} \beta_{(u) \nu \nu \mu}^{*}+4 \beta_{(u) \lambda \mu \mu} \beta_{(u) \lambda \nu \nu}^{*}+8 \beta_{(u) \lambda \mu \nu} \beta_{(u) \lambda \mu \nu}^{*}\right. \\
& \left.+\beta_{(u) \lambda \mu \mu} \beta_{(u) \nu \nu \lambda}^{*}+2 \beta_{(u) \lambda \mu \nu} \beta_{(u) \mu \nu \lambda}^{*}\right) .
\end{aligned}
$$

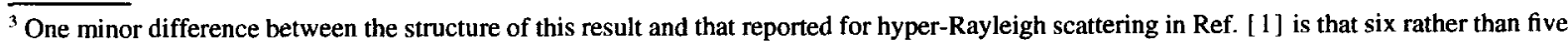
molecular invariants appear here. This reflects only the extension of the present theory to accommodate near-resonance behaviour: off-resonance, where the $\beta$ tensor is real, the result reduces precisely to the five-parameter form.
} 
Using circularly polarised pump radiation and resolving the harmonic for a component with the same helicity (both left-handed for example) we find

$$
\begin{aligned}
& \mathscr{J}^{\mathrm{SHG}}(\mathrm{L} \rightarrow \mathrm{L})=\frac{M \mathscr{D}^{(2)}}{105}\left(-2 \beta_{(u) \lambda \lambda \mu} \beta_{(u) \mu \nu \nu}^{*}+10 \beta_{(u) \lambda \lambda \mu} \beta_{(u) \nu \nu \mu}^{*}-\beta_{(u) \lambda \mu \mu} \beta_{(u) \lambda \nu \nu}^{*}+5 \beta_{(u) \lambda \mu \nu} \beta_{(u) \lambda \mu \nu}^{*}\right. \\
& \left.-2 \beta_{(u) \lambda \mu \mu} \beta_{(u) \nu \nu \lambda}^{*}-4 \beta_{(u) \lambda \mu \nu} \beta_{(u) \mu \nu \lambda}^{*}\right),
\end{aligned}
$$

and for a harmonic component with opposite helicity

$$
\begin{aligned}
& \mathscr{I}^{\mathrm{SHG}}(\mathrm{L} \rightarrow \mathrm{R})=\frac{M \mathscr{D}^{(2)}}{105}\left(-2 \beta_{(u) \lambda \lambda \mu} \beta_{(u) \mu \nu \nu}^{*}-4 \beta_{(u) \lambda \lambda \mu} \beta_{(u) \nu \nu \mu}^{*}-\beta_{(u) \lambda \mu \mu} \beta_{(u) \lambda \nu \nu}^{*}+5 \beta_{(u) \lambda \mu \nu} \beta_{(u) \lambda \mu \nu}^{*}\right. \\
& \left.-2 \beta_{(u) \lambda \mu \mu} \beta_{(u) \nu \nu \lambda}^{*}+10 \beta_{(u) \lambda \mu \nu} \beta_{(u) \mu \nu \lambda}^{*}\right) .
\end{aligned}
$$

In this case the experimentally observed harmonic intensity will be the combination of Eqs. (24) and (25):

$$
\begin{aligned}
& \mathscr{F}_{\mathrm{tot}(\mathrm{circular})}^{\mathrm{SHG}}=\frac{M \mathscr{D}^{(2)}}{105}\left(-4 \beta_{(u) \lambda \lambda \mu} \beta_{(u) \mu \nu \nu}^{*}+6 \beta_{(u) \lambda \lambda \mu} \beta_{(u) \nu \nu \mu}^{*}-2 \beta_{(u) \lambda \mu \mu} \beta_{(u) \lambda \nu \nu}^{*}+10 \beta_{(u) \lambda \mu \nu} \beta_{(u) \lambda \mu \nu}^{*}\right. \\
& \left.-4 \beta_{(u) \lambda \mu \mu} \beta_{(u) \nu \nu \lambda}^{*}+6 \beta_{(u) \lambda \mu \nu} \beta_{(u) \mu \nu \lambda}^{*}\right) .
\end{aligned}
$$

A key feature of the result (26), immediately open to experimental verification, is that harmonic emission should be sustained under conditions where the pump radiation is circularly polarised. This feature uniquely identifies the mechanism under discussion. Any coherent signal resulting from the normal mechanism for coherent harmonic emission (as for example that which might be associated with the intensity contribution $\mathscr{I}_{2}$ ) vanishes entirely if circularly polarised pump radiation is employed [24]; moreover the same embargo on the harmonic conversion of circularly polarised radiation applies to higher-order six-wave mixing mechanisms [6].

\section{Discussion}

By properly formulating the detailed procedure for dealing with the necessary orientational averages, the present theory reveals that the emergence of a second harmonic from a system of randomly oriented species such as a suspension can be understood in terms of a quasi-second harmonic generation having features both of second harmonic generation and hyper-Rayleigh scattering. The reason for the signal (16) being significantly larger for emission in the forward direction entails constructive interference between harmonic conversion events at different sites within each suspended particle, as in Eq. (14). In the experimental study which led to observation of the phenomenon, it is possible to identify several factors that contribute to the size of the signal.

First, it may be established that the coherence length for the harmonic conversion process is considerably larger than the size of each PM unit. Although often overlooked, incidental account should here be taken of the fact that the refractive indices of the disperse phase and the dispersion medium may have rather different values. According to Song et al. [12] the difference $\Delta n=n_{1064}-n_{532}=0.01$ for water and according to Huang and Lewis [25] the value for the dried membrane could be accepted as $\Delta n=0.005$. The first value leads to a coherence length of around $25 \mu \mathrm{m}$ and the second, $50 \mu \mathrm{m}$. It is to be supposed that the value for the membrane in aquo lies somewhere between these two values. The $1 \mu \mathrm{m}$ size of the PM units studied in Ref. [12], each of which contains tens of thousands of BR cells, is of the same order as the wavelength of incident laser light, $\lambda=1.064 \mu \mathrm{m}$, and much smaller than the coherence length and the laser beamwidth. Consequently fully constructive interference can take place within each unit.

In this connection, as mentioned in Section 3, it is significant that the second harmonic signal scales linearly with $M$, the number of units, but quadratically with $n$, the number of chromophore sites within each unit. In essence this means that the forward harmonic emission from the system is larger by a factor of $n$ than the signal that would be 
produced by the same number of molecules without any structural correlation. In each unit the BR molecules are highly oriented, while individual units in the solvent are randomly distributed and oriented. The theory developed above would thus suggest that, on this basis alone, the signal should be at least $10^{4}$ times larger than a solution of the same number of randomly oriented and uncorrelated BR molecules. The lower limit on the number of molecules per unit that could lead to an observable signal will in practice be determined by the degree to which the detection system is geared to discriminate against background hyper-Rayleigh scattering.

Returning specifically to the purple membrane results, a further reason for the size of the response concerns the electronic structure of bacteriorhodopsin. It is well known that the chromophore within BR is a retinal, bound to lysine via a protonated Schiff base linkage [13]. The spectral response while the PM is in the states $\mathrm{K}$ or $\mathrm{L}$ centres on approximately 550-610 $\mathrm{nm} \mathrm{[26];} \mathrm{the} \mathrm{input} \mathrm{laser} \mathrm{radiation} \mathrm{is} \mathrm{thus} \mathrm{close} \mathrm{to} \mathrm{resonance} \mathrm{with} \mathrm{the} \mathrm{second} \mathrm{harmonic}$ signal, and as such the response is dominated by two-level behaviour. It has been shown that such a process may be driven by a second harmonic susceptibility that is dependent upon the difference between the static electronic dipole moments of the resonant and ground states. $[27,28]$ In fact the retinal chromophore has been shown to have an unusually large difference between its ground and excited state dipole moments [29] and as such will undoubtedly increase the magnitude of the hyperpolarisability. A secondary enhancement factor is thus associated with the combination of a resonant enhanced transition and the large dipole moment shift exhibited by the chromophore.

In summary, the theory developed here provides a full rationalisation for the second harmonic signals observed by Song et al. [12]. The framework of the theory suggests that similar observations might be made in other randomly oriented systems wherein there is a degree of local orientational correlation, as may be the case in many liquids. However the size of the harmonic signal depends crucially on the number of molecules within each unit that may be regarded as correlated, and this is undoubtedly one reason for the remarkable results obtained with bacteriorhodopsin. Another factor to be taken into consideration is the driving force afforded by the ground and excited state dipole difference. Our theory leads to the prediction that the second harmonic will remain measurable when a quarter-wave plate is inserted in the laser beam, so as to produce circularly polarised pump radiation. Verification of the mechanism will open a number of avenues for further study of this phenomenon which clearly has significant implications, not least the validity of employing SHG as a surface-selective probe.

\section{Acknowledgements}

We are pleased to acknowledge support from the Engineering and Physical Sciences Research Council (PA) and the Royal Society (AAD).

\section{References}

[1] D.L. Andrews, Advan. Chem. Phys. 85 (1993) 545.

[2] S. Kielich, IEEE J. Quantum Electron. QE-5 (1969) 562.

[3] S. Kielich, Opto-electronics 2 (1970) 5.

[4] S. Kielich, in: Nonlinear behaviour of molecules, atoms and ions in electric, magnetic or electromagnetic fields, ed. L. Neel (Elsevier, Amsterdam, 1979) p. 111.

[5] D.L. Andrews and B.S. Sherborne, J. Phys. B 19 (1986) 4265.

[6] D.L. Andrews, Nonlin. Opt. 8 (1994) 25.

[7] U. Österberg and W. Margulis, Opt. Letters 11 (1986) 516.

[8] M.C. Farries, P. St. J. Russell, M.E. Fermann and D.N. Payne, Electron. Letters 23 (1987) 322.

[9] R.H. Stolen and H.W.K. Tom, Opt. Letters 12 (1987) 585.

[10] V. Mizrahi, Y. Hibino and G. Stegeman, Opt. Commun. 78 (1990) 283.

[11] V. Dominic and J. Feinberg, Opt. Letters 17 (1992) 1761.

[12] Q. Song, C. Wan and C.K. Johnson, J. Phys. Chem. 98 (1994) 1999.

[13] D. Oesterhelt and W. Stoeckenius, Nature New Biol. 233 (1971) 149. 
[14] R. Henderson, J.M. Baldwin, T.A. Ceska, F. Zemlin, E. Beckmann and K.H. Downing, J. Mol. Biol. 213 (1990) 899.

[15] W. Humphrey, I. Logunov, K. Schulten and M. Sheves, Biochemistry 33 (1994) 3668.

[16] R.A. Mathies, S.W. Lin, J.B. Ames and W.J. Pollard, Ann. Rev. Biophys. Biophys. Chem. 20 (1991) 491.

[17] R.R. Birge and C.-F. Zhang, J. Chem. Phys. 92 (1990) 7178.

[18] S. Kielich, in: Progress in optics, Vol. 20, ed. E. Wolf (North-Holland, Amsterdam, 1983) p. 157.

| $19 \mid$ C.H. Wang, J. Chem. Phys. 98 (1993) 3457

[20] D.L. Andrews, J. Phys. B 13 (1980) 4091.

|21] P.D. Maker, Phys. Rev. A 1 (1970) 923.

[22] D. Marcuse, Principles of quantum electronics (Academic Press, New York, 1980).

123| D.L. Andrews and T. Thirunamachandran, J. Chem. Phys. 67 (1977) 5026.

124] C.L. Tang and H. Rabin, Phys. Rev. B 3 (1971) 4025.

[25| J.Y. Huang and A. Lewis, Biophys. J. 55 (1989) 835.

[26] C. Wan, J. Qian and C.K. Johnson, Biochemistry 30 (1991) 394.

[27] J.L. Oudar and D.S. Chemla, J. Chem. Phys. 66 (1977) 2664

[28] D.L. Andrews and W.J. Meath, J. Phys. B 26 (1993) 4633.

[29] R. Mathies and L. Stryer, Proc. Natl. Acad. Sci. USA 73 (1976) 2169 